\title{
ASO Author Reflections: A Novel Three-IncRNA Signature Predictive of Prognoses of HNSCC Patients
}

\author{
Hongyi Jiang, MM ${ }^{1,2}$, Ben Ma, MD ${ }^{1,2}$, Weibo Xu, MM ${ }^{1,2}$, Yi Luo, MM ${ }^{1,2}$, Zhongwu Lu, MD ${ }^{1,2}$, \\ Tian Liao, MM ${ }^{1,2}$, Xiao Wang, $\mathrm{MD}^{1,2}$, Shishuai Wen, $\mathrm{MD}^{1,2}$, Shuwen Yang, $\mathrm{MD}^{1,2}$, and Yu Wang, $\mathrm{MD}^{1,2}$ \\ ${ }^{1}$ Department of Head and Neck Surgery, Fudan University Shanghai Cancer Center, Shanghai, China; ${ }^{2}$ Department of \\ Oncology, Shanghai Medical College of Fudan University, Shanghai, China
}

Recently, the prognostic role of long noncoding RNAs (lncRNAs) has been increasingly noted in various cancer types including head and neck squamous cell carcinoma (HNSCC). However, the published work is mostly limited to public databases, lacking an independent cohort to further validate the utility of the identified lncRNA signatures.

In this study, we identified several disease-associated lncRNAs from The Cancer Genome Atlas (TCGA) dataset to develop an independent prognosis prediction model and further validated the utility of identified lncRNA signatures in cases from our center, the Fudan University Shanghai Cancer Center (FUSCC).

Though the three lncRNAs were observed to be a potent biomarker for prognosis, the related mechanisms in HNSCC have not yet been clarified. It will be intriguing to investigate the biological roles of aberrant molecular pathways caused by these three IncRNAs in $\mathrm{HNSCC}^{1-5}$.

\section{REFERENCES}

1. Nohata N, Abba M, Gutkind JS. Unraveling the oral cancer lncRNAome: identification of novel lncRNAs associated with malignant progression and HPV infection. Oral Oncol. 2016;59:58-66.

2. Cao W, Liu J, Liu Z, Wang X, Han Z, Ji T, et al. A three-lncRNA signature derived from the Atlas of ncRNA in cancer (TANRIC) database predicts the survival of patients with head and neck squamous cell carcinoma. Oral Oncol. 2017;65:94-101.

3. Liu G, Zheng J, Zhuang L, Lv Y, Zhu G, Pi L, et al. A prognostic 5-lncRNA expression signature for head and neck squamous cell carcinoma. Sci Rep UK. 2018;8:15250.

4. Zhang Z, Zhao L, Chai L, Zhou S, Wang F, Wei Y, et al. Seven LncRNA-mRNA based risk score predicts the survival of head and neck squamous cell carcinoma. Sci Rep UK. 2017;7: 309.

5. Jiang H, Ma B, Xu W, Luo Y, Wang X, Wen S, Liao T, Lu Z, Yang S, Wang Y. A novel three-lncRNA signature predicts the overall survival of HNSCC patients. ANSO. 2020. https://doi.org/ 10.1245/s10434-020-09210-1.

Publisher's Note Springer Nature remains neutral with regard to jurisdictional claims in published maps and institutional affiliations.

(C) Society of Surgical Oncology 2020

First Received: 25 September 2020

Accepted: 27 September 2020;

Published Online: 16 November 2020

H. Jiang, MM

e-mail: 18211230013@fudan.edu.cn 